\title{
Guiding Manufacturing Enterprises to Achieve Long-Term Business Sustain- ability Using the Collective System Design Approach
}

\author{
Shahab A. Shah ${ }^{1, *}$, Joseph J. Smith ${ }^{1}$, and David S. Cochran ${ }^{1}$ \\ ${ }^{1}$ Purdue University Fort Wayne, Fort Wayne, IN 46835 United States
}

\begin{abstract}
This paper addresses several issues discovered by working with different industries attempting to sustain their business enterprise and to become "Lean." The three key points addressed by the paper are: 1) Designing a new system or re-designing an existing system should focus on collecting a complete set of customer needs and deriving functional requirements from those needs. 2) Illustrating the path-dependency or sequence of implementation of Physical Solutions (PSs) to achieve Functional Requirements (FRs) of partially coupled designs. 3) Lean is not what we implement as a system, rather lean is what we become as a result of meeting customer needs with the utilization of the least amount of resources possible in a sustained manner. When lean is viewed as a set of tools to implement, the people in an enterprise will face ever-increasing difficulty in long-term business sustainability. The primary problems arise early in the design/re-design phase due to the lack of a clear set of system functional requirements. Without clearly defined system FRs, driven by recognizing customer needs/concerns, an enterprise will implement point solutions in an attempt to improve part(s) of a system. The Manufacturing System Design Decomposition (MSDD), a product of Axiomatic Design (AD), illustrates the path-dependency among the solutions of the associated requirements of any manufacturing facility. The MSDD provides a system-wide view and a clear sequence for system design implementation. The Collective System Design approach is discussed to provide the steps for senior leadership to re-design an existing system or to design a new system that results in long-term sustainability and become "lean."
\end{abstract}

\section{Introduction}

The aim of this paper is to offer an approach to manufacturing system design and to "become lean" and provide the long-term business sustainability using the Collective System Design approach.

The term "lean" was first coined by J. Krafcik in 1988 [1], the reference was to a system conceived in the 1950s and is known as the Toyota Production System (TPS) [2]. The major contributors to TPS were Taichii Ohno, Eiji Toyoda, and Shigeo Shingo at the Toyota Motor Corporation, Japan [2]. Masaki Imai claimed in 1986 that Toyota came up with the idea of a system in which small quantity and a large variety of many different vehicles are manufactured using a tightrope of inventory, which eventually became the reason behind Toyota's success [3].

There are many different definitions of lean [4], but early on, lean was misunderstood to be the elimination of waste from the system [5]. Later on, lean meant "cutting heads" and positions, resulting in part shortages as lean meant zero inventory $[6,7]$. Womack, Jones, and Roos introduced the term "lean production" to the Western world to describe the TPS which defines a lean system as a system that prioritizes the improvement of the flow of product/service to the customer without any non-value added activities by integrating the best aspects of craft production with those of mass production $[2,8]$.

\footnotetext{
*e-mail: shahab.alishah05@gmail.com
}

The lean ideology is growing popular in both the manufacturing and service sector. However, most industries suffer in getting the right understanding of the lean ideology of satisfying the customer needs. These industries consider lean to be a toolbox which can be implemented in an enterprise [9]. Some industries consider lean implementation to be a package of several tools such as $5 \mathrm{~S}$ (Sort, Set in order, Shine, Standardize, and Sustain), Single Minute Exchange of Die (SMED) visual control, pokayoke (i.e., mistake-proofing), Just in Time (JIT), Heijunka Box (production leveling), continuous improvement, etc. [10-12]. All these tools help improve parts of the system rather than enhancing the system-wide outcome.

A lean system is not a toolbox or an activity that can be implemented. Implementation of a tool without knowing the real purpose behind its selection results in unknown consequences and the lack of sustainability. Rather, lean can be defined as, "the result of a system design that is tailored to meet customer needs." Meeting customer needs involves eliminating non-value added activities by employing the right tone and by understanding the pathdependency of implementing solutions within the system design [13-15].

The CSD approach enables an enterprise team to develop a long-term enterprise design that is sustainable using AD. This paper illustrates the CSD approach and an application with a commercial vehicle manufacturer. 


\section{Literature Review}

According to the literature, only less than $10 \%$ of the industries that implement lean are able to sustain the system design after three years $[13,14,16]$. The question arises that if all the tools that can help a system to become lean are available, what is missing to make the use of these tools sustainable [17]?

There are numerous studies and quotations regarding the importance of positive attitude in a system towards becoming lean. For example, a keynote speaker at the 2014 Oklohama conference stated that a system needs to have the presence of two factors (tools and culture) with the following proportion in order to become lean:

1. Implement the necessary tools which are about $40 \%$ of the total effect.

2. Develop the people in the system with positive attitude, which is about $60 \%$ of the total effect [18].

In addition, Dr. Deming said " $94 \%$ of the problems in business are system driven and only $6 \%$ are people driven" which captures the importance of positive tone [19].

Numerous industries employ the various tools used by Toyota (i.e., 5S, producing to takt time), but often fail to achieve the desired outcomes. These tools represent the "how," but without knowing the "why," the tools will be misused. The problem with not understanding the why before implementing the how is a characteristic of many different industries including agriculture [20]. The culture of traditional management accounting is to jump for the tools and solutions followed by TPS rather than knowing the real intent for which the tool is used [21]. As such, an understanding of the motivation and the design intent behind using a tool plays a vital role in bringing the success of the function provided by the tool.

Once the intent behind the selection of a tool for a requirement is known, the enterprise needs to know the sequencing of operations. That is, which operations come first before the implementation of the chosen tool. In other words, understanding the system as a whole and not trying to improve parts of the system should be the central focus of interest. According to Taiichi Ohno, "The key to the Toyota Way and what makes Toyota stand out is not any of the individual elements. But what is important is having all the elements together as a system. It must be practiced every day in a very consistent manner, not in spurts [22]."

The lack of understanding in the manufacturing system design is analogous to the lack of understanding of medication interactions and the resulting adverse effects on the patients' health. For instance, a patient who is on four or more medications has an increased risk of suffering from adverse drug reactions, which results from a lack of understanding of the system as a whole [23].

According to Taiichi Ohno, "Where there is no Standard there can be no Kaizen (continuous improvement) [24]." Therefore, it is very important to develop a standard work for implementing the chosen solution and look for opportunities for improvement by changing either the FRs or the PSs or both $[19,25]$.

\section{Approach}

Collective System Design (CSD) is an enterprise system design approach that Dr. David S. Cochran began developing during the mid-2000's. CSD is the practice of the System Engineering Center that Dr. Cochran directs at Purdue University, Fort Wayne [26].

\subsection{CSD Language}

The CSD language employs the terminology of Functional Requirements (FRs) and Physical Solutions (PSs), that aims to segregate what the system must achieve (FRs) from how to achieve (PSs), adapted from $\mathrm{AD}$ [26, 27], as shown in Fig. 1.

The terminology used for the mean(s) of achieving a Functional Requirement (FR) in the traditional Axiomatic Design is "Design Parameter (DP)" [27], which has been re-named/updated to "Physical Solution (PS)" in the CSD approach for two reasons:

1. The physical domain in the design framework of axiomatic design expresses a physical thing/object that is proposed to achieve the functional requirements stated within the functional domain.

2. Many technical and most non-technical professionals may not be familiar with the term, "Design Parameters," and may lack the connection that to achieve an FR, it requires the selection of a PS as the "How" to achieve an FR. The PS acknowledges that we are choosing a physical thing (noun) to achieve an FR that starts with a verb.

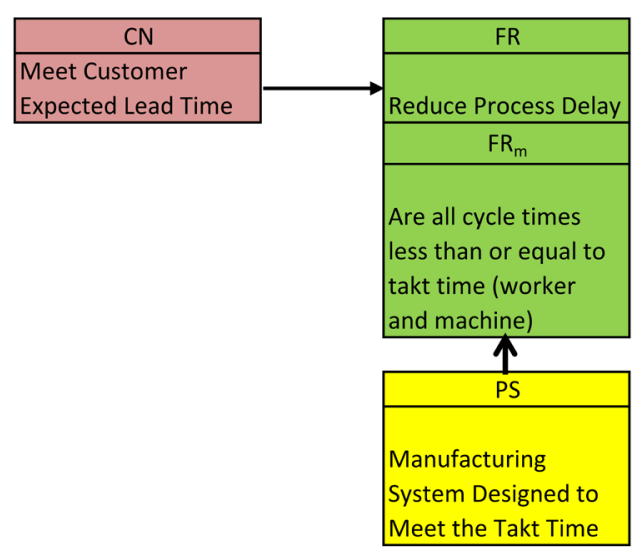

Figure 1. CSD Representation of FR

\subsection{General Overview of CSD: The Flame Model}

CSD is a 12-step design approach for designing new and improving the existing systems with a focus on sustainability of system designs to meet the customer needs. Step 1-3 covers the tone and culture of the senior leadership, step 4-7 covers the thinking part of the enterprise for meeting the customer needs which is based on Axiomatic Design principles, step 8 covers the structure, step 9 covers 
the plan for continuous improvement, and step 10-12 covers the feedback for sustainability and growth of the enterprise [28]. No improvement can be sustained in an enterprise if the feedback for sustainability steps are not part of the tone of the people in the enterprise. In this paper, the flame model provides a general overview of CSD approach, as shown in Fig. 2.

\subsection{Elements of the Flame Model}

The flame model represents a hierarchy of the different elements that make up systems namely, tone, thinking, structure, and work/actions. These elements are expressed as different layers cohesively connected like the different layers within a flame. All these elements are present within any system at any given time. The outcome of each layer is either formed intentionally or becomes unintentional due to the lack of attention given to that associated element. The work/actions and structure are the physical elements that can easily be seen, however, the thinking and tone are the hidden elements [30].

Diagnosis of a problem and design (or redesign) of a system are both expressed by the flame model.

\subsection{Diagnosis Phase}

The diagnosis phase looks at determining the root cause of the problem by first understanding the work and actions (this layer is often referred to as going to the Gemba) [31]. After determining the root cause, it is important to understand that whether the problem arises due to the operator's fault or due to the system's fault. That's why the structure of the system that drives the work and actions is understood next. The succeeding element is the thinking layer of the system and this is where FRs and associated PSs are defined. The thinking layer is where people may or may not know what the FRs are or people do or do not collectively agree on those FRs. And the last element is the tone, which represents the attitude of the people, or what many refer to as the culture of the system [32].

\subsection{Design Phase}

\subsubsection{Tone}

Once the problem is diagnosed and a conscious choice to change is made by the senior leadership, the system design can be realized by moving outward through the layers of the flame. A proper leadership tone is required for meeting the needs of the internal customer (people working inside the enterprise) resulting in a high working morale of the internal customers which would indirectly help achieve the needs of the external customers (end customers). A proper tone basically facilitates a respectful environment that allows both the direct and indirect people to be engaged [5], and the understanding that a system should not let the people fail but rather keeps them from failing [19]. Recognizing the failure of a system is an opportunity for continuous improvement in the system that leads to the sustainability of the system design.

\subsubsection{Thinking}

This tone of conscious decision making by the leadership leads to the Manufacturing System Design Decomposition (MSDD)' focus on meeting all the customer needs, as shown in Fig. 3, illustrates the thinking layer of the CSD Flame Model [32]. The MSDD is developed with the first axiom of axiomatic design theory [26].

This decomposition is a translation of all the external and internal customer needs of a manufacturing system to become lean and to achieve the long-term sustainability of the system design using the language of CSD. Whereas, external needs refer to the needs of the end customer and internal needs refer to the needs of the people working inside the enterprise.

Overall, the requirements in the MSDD can be grouped into six key areas: Quality, Identifying and Resolving Problems, Predictable Output, Delay Reduction, Operation (direct and indirect labor) Costs, and Investment [34]. These six key areas consist of a total of seventy FRPS pairs, typically used in manufacturing.

The MSDD represents an adaption of Axiomatic Design theory. By maintaining the independence of the FRs (derived from customer needs), the path-dependency from left to right of the design relationships within a manufacturing facility can be realized as shown in Fig. 3 [27]. For example, Quality branch impacts all the branches to the right in a manufacturing system, based on the proposed MSDD.

The design relationship matrix for level III of MSDD, showing the partially dependent design, is shown in Eq. 1.

$$
\left(\begin{array}{l}
F R 111 \\
F R 112 \\
F R 113 \\
F R 121 \\
F R 122 \\
F R 123
\end{array}\right)=\left[\begin{array}{llllll}
X & 0 & 0 & 0 & 0 & 0 \\
X & X & 0 & 0 & 0 & 0 \\
X & X & X & 0 & 0 & 0 \\
X & X & X & X & 0 & 0 \\
X & X & X & X & X & 0 \\
X & X & X & X & X & X
\end{array}\right] \times\left(\begin{array}{l}
P S 111 \\
P S 112 \\
P S 113 \\
P S 121 \\
P S 122 \\
P S 123
\end{array}\right)
$$

To simplify the communication of the MSDD to the people within a manufacturing enterprise, the seven teaching FRs were developed:

FR1: "Provide a safe, healthy environment,"

FR2: "Produce the customer consumed quantity every shift,"

FR3: "Produce the customer consumed mix every shift,"

FR4: "Do not advance a defect to the next customer of your work,"

FR5: "Achieve FR1 through FR4 in spite of variation,"

FR6: "Rapidly identify problems and resolve them for the long-term," and

FR7: "Achieve FR1 through FR6 with the least time in system," 


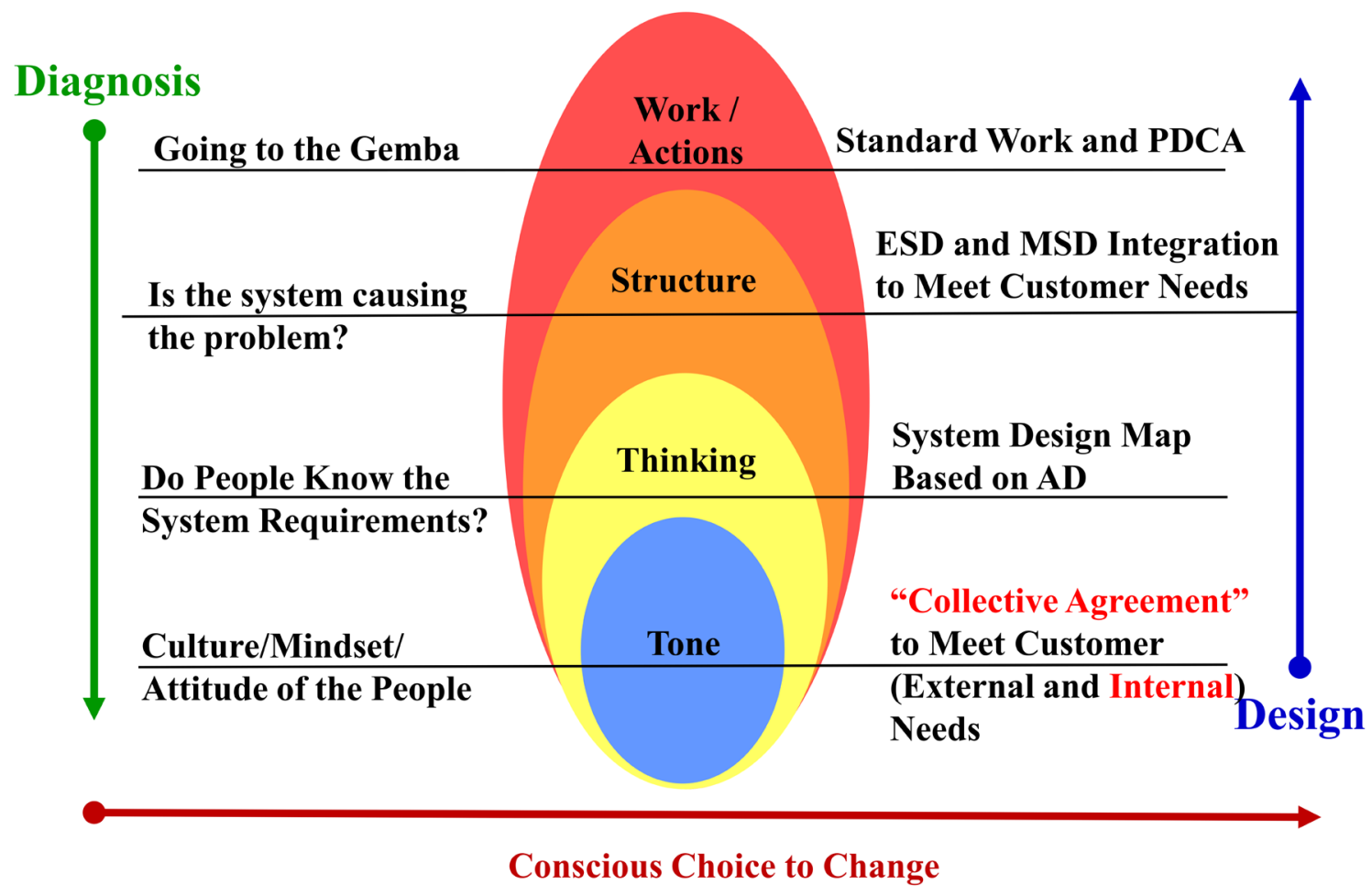

Figure 2. Flame Model of CSD. Updated from [29]

Within the seven teaching FRs (see Fig. 3), FR1: "Provide a safe, healthy environment," is depicted to be at the very left, indicating its criticality and that it impacts all the rest of the FRs. However, due to the liability associated with plant safety, the safety branch was not included on the MSDD. Also, the PSs are intentionally left blank in the teaching FRs of MSDD to be filled up by the users with collective agreement among the team members. Since the path-dependency of FR-PS pairs is based on the PS selection, no dependencies are yet drawn for the teaching FRs.

\subsubsection{Structure}

The structure of an enterprise determines how the stations are laid out in the workplace and who reports to who. According to CSD, the Manufacturing System Design (MSD) and Enterprise System Design (ESD) need to work together to sustain the system design that helps achieve customer needs, as shown in Fig. 4. Whereas, ESD contains Engineering Director (ED), Quality Director (QD), Finance and Accounting (F \& A), Management (M), Human Resource (HR), Sales (S), and Controller (C) etc. And the MSD consists of value stream mapping, process mapping, machines, and operators etc. to produce to the customer needs. For instance, a product design team may develop a design for a product with a very good quality (low tolerance) but if the resources (machines, operators, or layout etc.) are unable to produce a product to the design specifications so all the efforts of the design team will go in vain and vice versa.

\subsubsection{Work/Actions}

Work/Actions refers to defining and implementing the standard work methods. The standard work may include the Process Variables (PVs) but normally devise the procedure to implement a PS. For instance, if a chef is backing the cake so the whole procedure is devised through work/actions but setting the parameters like the temperature of the cake etc. can be the PVs.

Standard work methods should be defined based on the current best-known methods to be improved in the future as new methods are known, as opposed to planning for perfection. These different layers of the CSD flame model are utilized in the next sections to sustain the long-term business system design and to become lean.

\section{Becoming Lean using CSD Approach}

\subsection{Leadership Behavior}

The CSD approach begins by framing the problem that needs to be overcome, in order to understand the stakeholders of the system. This approach fosters an environment where the stakeholders work together as a team 


\section{Manufacturing System Design Decomposition (MSDD)}

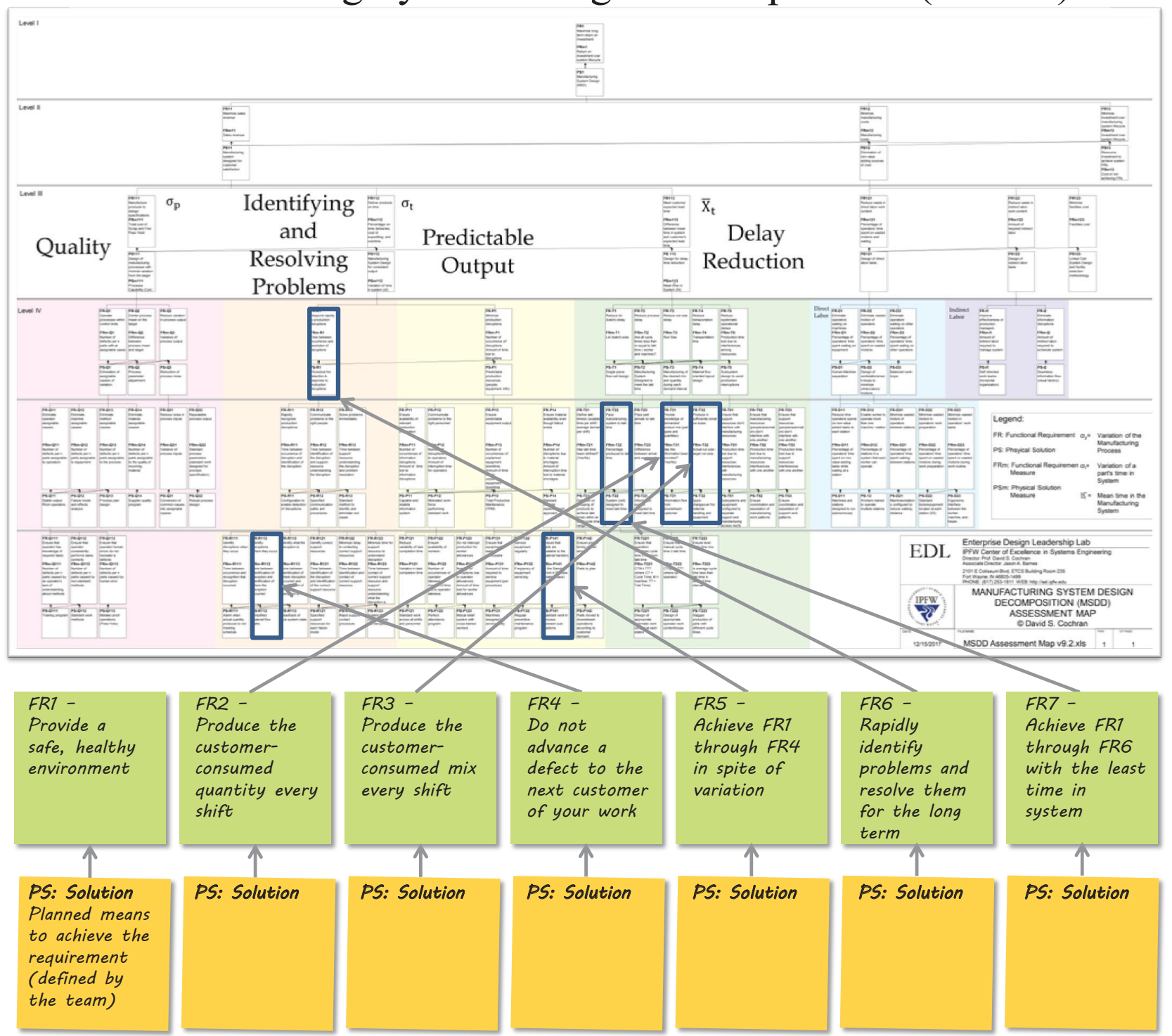

Figure 3. Manufacturing System Design Decomposition. Updated from [33]. For detailed observation of the individual FR-PS pair, Zoom-in on the figure in the digital copy.

and make a conscious choice to change in the system. These days, most of the manufacturing industries either run into overproduction waste or lack of enough production to meet the customer needs leading to sub-contracting. Besides, the industries also usually suffer from being flexible enough to offer product customization based on varying customer needs [35]. Therefore, in this paper, change in the system is indicating the redesign of the system to become lean. i.e., to meet the customer consumed quantity and mix of good quality parts every shift with the consumption of the least amount of resources possible.

\subsection{Collective Agreement on FRs, driven by Customer Needs}

Once the leadership team makes a conscious decision to become lean, the enterprise must collectively agree on determining the FRs of the system before choosing the PSs.
Rather than just replicating an idea/tool employed by TPS without understanding the intent (FR) of the tool (PS). For instance, TPS has chosen the PS: "Manufacturing System Designed to Takt Time" to achieve the FR: "Reduce Process Delay" to help assist in meeting the customer expected lead time with the least amount of non-value added activities [36].

Takt time synchronizes the production flow of each shift to the average customer demand desired per shift. Mathematically, takt time is defined in Eq. 2 [30]. Takt time sets the pace for production by limiting the maximum cycle time of the line. Takt time represents the time available for the system to produce a single part, measured in mins/part.

$$
\text { Takt Time }=\frac{\text { Available Time per Shift }}{\text { Average Customer Demand per Shift }}
$$




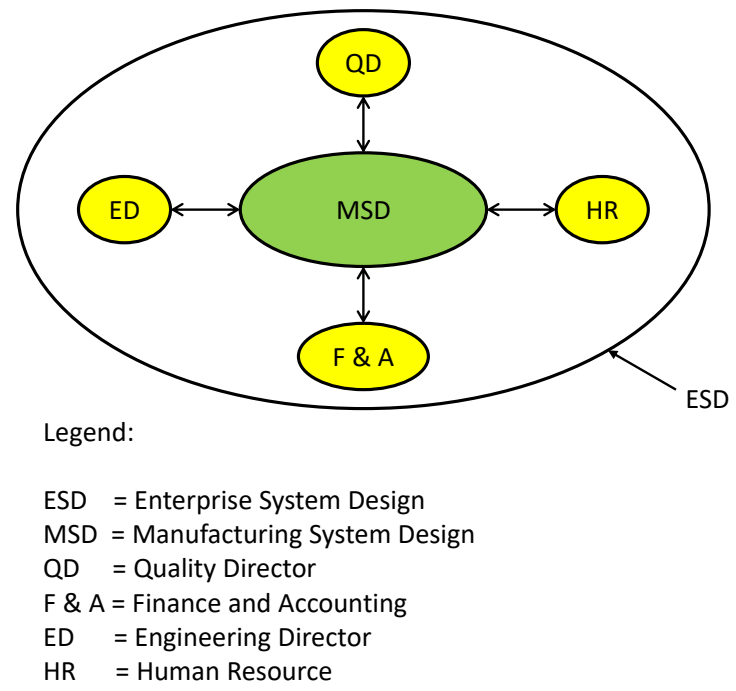

Figure 4. Integral Structure of MSD and ESD

However, one of the industry clients has replicated the PS: "Manufacturing System Design to Meet the Takt Time" from TPS with a lack of understanding of the design intent. Choosing the PS without a conscious understanding of the FR: "Reduce Process Delay" leads to certain undesirable behaviors such as producing to the minimum cycle time (produce as fast as possible). That's why the tool is just simply used for checking the monthly numbers rather than producing to the customer expected lead time (intent).

CSD, the proposed approach, addresses this issue of lack of understanding of the customer need or intent behind implementing a tool by understanding the customer need $(\mathrm{CN})$ first, followed by FR-PS pair derivation as shown in Fig. 1. This approach of implementing a tool in manufacturing system would compel the management to have a clear and an accurate understanding of the customer need.

\subsection{Precedence Order of Implementation of PSs}

After understanding the purpose/intent of a tool, the precedence/sequential order of implementation of the PSs to achieve the associated FRs plays a crucial role in providing a strong foundation for lean.

For instance, an industry could set the precedence order of tools by asking a question, "Does the PS1 affect the achievement of FR2?" If the PS1 does affect the achievement of FR2, so the indication is to proceed to PS2 (used for achieving FR2) after PS1 implementation. However, if the PS1 does not affect the achievement of FR2, then there is no precedence order between the FR1-PS1 pair and FR2-PS2 pair.

In the current example of choosing the PS: "Manufacturing System Designed to Takt Time," the industry has to improve the quality first to ensure production of good parts to takt time. If the cell is designed to takt time and there are repetitive quality failures so the system may produce parts to the customer consumed quantity but after removing the rejected parts, the production to takt time will not be ensured. Next, the industry has to have a procedure for detection and response to production disruptions by employing many different tools. e.g., an alarm system would sound in the management offices or any other alert system for identifying disruptions when the actual quantity produced is not meeting the schedule etc. Next, the manufacturing system designed to have a standard work defined for achieving predictable production resources (people, equipment, information) leading to minimizing the production disruptions. Next, the cells/stations should be designed to single-piece flow for achieving the FR: "Reduce lot (batch) delay."

Once all these tools/solutions are implemented following a precedence order, that's when the manufacturing system can be designed to takt time to help produce to customer expected lead time. In the similar fashion, if all the FR-PS pairs of MSDD, as shown in Fig. 3, that apply to a manufacturing enterprise is being implemented knowing the effect on the customer needs, the enterprise will result in becoming lean.

\section{Sustaining the Long-Term Business Sustainability}

A system can attempt to employ two different approaches to sustain itself while meeting the customer needs in the long run: (1) Implement a single-step, point solutions approach and (2) CSD approach, i.e., decompose the highestlevel PS into the lowest (leaf)-level FR-PS pairs that can be implemented easily as shown in Fig. 5 and Fig. 6.

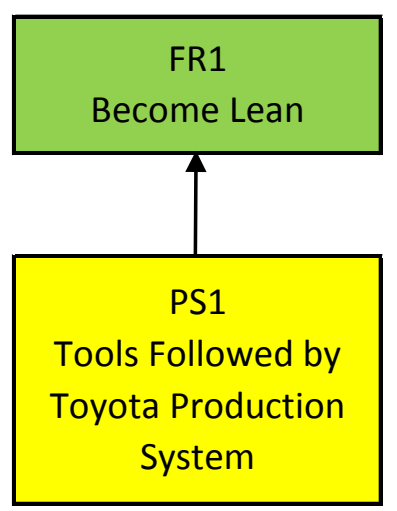

Figure 5. Single-Step, Point Solution Approach, Failing to Sustain the System Design

\subsection{Single-Step Approach vs. CSD Approach to Sustain the System Design}

In the single-step approach, the system attempts to achieve only a single FR by implementing a point solution. For instance, the system may or may not be able to achieve the FR, derived from the CN: "Meet customer consumed 


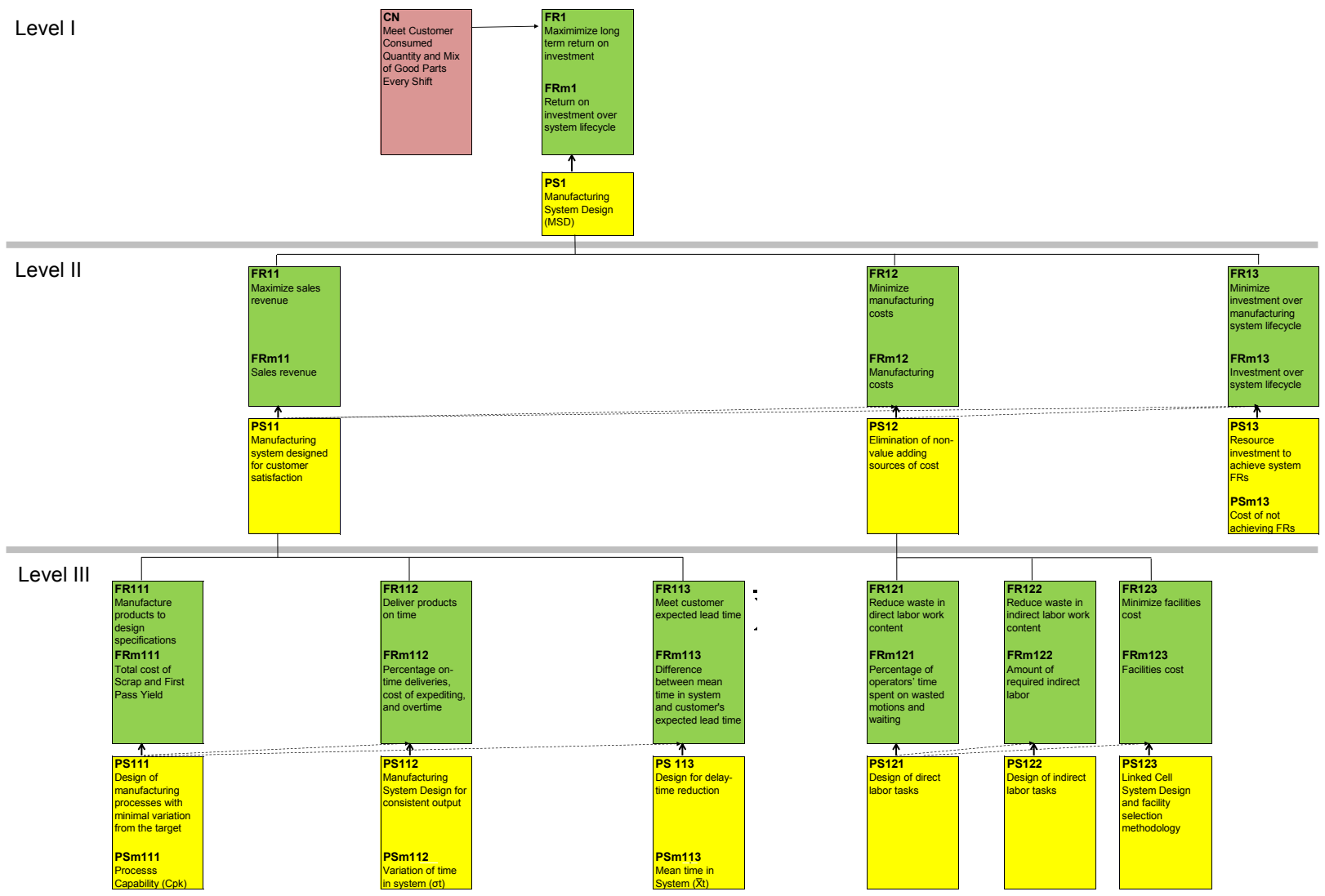

Figure 6. CSD Approach to Sustain the System Design

quantity and mix of good parts every shift" with the implementation of the chosen PS.

The trap that an industry usually falls into is that to become lean and sustain the system design, requires achieving all of the FRs of the system design simultaneously. This point illustrates that choosing only one solution does not ensure that an industry will become lean.

The CSD approach is to:

1. The design intent of the tool.

2. All the FR-PS pairs of the system design decomposition needed to achieve the top-level FR to achieve customer need.

3. The precedence order (partial-coupling) of the FRPS pairs.

Each FR-PS pair has associated measures, called FRm and PSm which indicates the success or failure. Examples of FRm are shown in Fig. 1 and Fig. 6 . A good measure for the PS should be in the form of "Yes" or "No" questions. i.e., the system either achieve what it was supposed to achieve or not with the implemented solution, rather than in the form of complicated mathematical algorithms and calculations. The decomposition clarifies the achievement of individual FRs in a sequential order using the implementation of associated PSs. If the system is showing a consistent increase in the system-wide outcome with the implementation of each leaf PS then the system is always approaching towards the highest-level FR. A system that becomes lean using this decomposition approach would be able to sustain the system design in a long-run due to both the readiness of process replication for developing standard work and the ease of continuous improvement.

\subsection{Standard Work}

Standard work is a key aspect of sustaining the system design to provide long-term business sustainability at any enterprise. Usually, industries end up with having a large buffer of inventory to meet the customer needs (quantity and mix) because the operations are not predictable. Standard work is a list of instructions that all the operators of each shift are trained to follow to provide a very repeatable, consistent, and predictable output. This predictable output would lead to tightening of the rope of inventory and a single-piece flow resulting in the reduction of the 5 different transportation delays (batch delay, process delay, run size delay, reduce transportation delay, and systematic operational delays) thereby reducing the associated cost measures as well, as shown in Fig. 3 [36]. Two types of standard work documents are important in a lean enterprise:

1. White sheet standard work details the day-in and day-out practices that need to be followed in a workplace and 
2. Green sheet standard work details the response plan for abnormal condition(s). i.e., visual management for identifying problems at their source (source inspection), and then resolving the problems immediately (control poka-yoke) etc. [37].

Consider the importance of standard work in the case of baking a cake. If a chef makes a cake by adding all the required ingredients without documenting the ratios of the ingredients and the environmental factors like temperature, humidity, etc, how will the process be repeatable? Maybe the cake comes out to be either dry, moist or even the best cake the customer has ever eaten. Upon request for another cake of the same shape and taste, the chef may never be able to replicate the baking process to result in the same kind of cake.

In order to replicate the process in a similar fashion every time, the chef has to document the instructions, detailing the ratio of ingredients, the timing, the temperature of the oven, and the environmental factors. This list of instructions would be known as white sheet standard work. In the case of baking a cake, green sheet standard work may be necessary when the chef forgets to grease the pan or for any other abnormality that may occur when baking a cake.

\subsection{Continuous Improvement}

In the CSD approach, after standard work is defined, the Plan-Do-Check-Act (PDCA) cycle is employed for continuous improvement. The PDCA cycle is aligned with the design relationships expressed by the MSDD, as shown in Fig. 7, to ensure that the requirements of the system are being met.

In the PDCA cycle, "Plan" refers to the development of standard work by collectively agreeing on the FRs of the manufacturing system design. Standard work also incorporates the proposal of PSs for achieving the associated FRs, along with measures of success defined for both the FRs and PSs. The standard work document associated with each station/department should be disseminated throughout the entire system with visual cues for easy reference.

"Do" refers to the implementation of the planned standard work in the enterprise. All of the stakeholders and employees are trained on the standard work documents to ensure everyone, even across different shifts, perform the work in the same manner. "Check" means to compare the physical outcomes of the implemented standard work against the defined measures of FRs and PSs. If the system outcome is in accordance with the measures defined for the FRs and PSs, then the proposed plan is accepted. Otherwise, an action needs to be taken to either change the PSs or/and in some cases even the FRs which leads to another iteration of the cycle for continuous improvement. The PS is changed if the FR is not achieved. However, the FR is changed if it is not correctly derived from a customer need, or in an event where the customer need changes itself [25].

In either of the two action scenarios described in PDCA cycle, training has to be provided based on the "up- dated" standard work to sustain the path of improvement for further iterations.

The analogy of baking a cake used earlier showed the importance of standard work, which is the basis for continuous improvement. Now if the FR for the chef is to bake a light and fluffy cake, the chef can add an extra ingredient (an egg etc.) to the cake (Plan), expecting an even softer cake while aiming for continuous improvement to exceed the customer satisfaction level. Once the cake is baked (Do), the chef would assess the cake against the order details provided by the customer (Check). If the cake does not turn out to be light and fluffy, the type or amount of ingredient needs to be changed (Act-Change PS). However, if the cake does turn out to be light and fluffy and is presented to the customer but the customer describes a different need other than a light and fluffy cake, that means the FR derived from the customer need(s) was wrong, and the recipe needs to be changed to the updated FR (Act-Change FR).

\section{Re-design of a Manufacturing System Based on the MSDD Evaluation and Simulation}

The innovation that CSD brings is the integration of understanding FRs and the leadership that is necessary for a team to collectively agree on and implement PSs. Therefore, CSD serves as both an approach for change leadership and a system design using AD.

MSDD can be used to evaluate/compare many different manufacturing system designs [38]. The HeatingVentilation-Air Conditioning (HVAC) small bus manufacturing system design at Mobile Climate Control (MCC), Goshen, IN. was chosen for evaluation on the basis of the MSDD, as shown in Fig. 8, highlights the opportunities for improvement in the system.

Based on the improvement opportunities dictated by the MSDD evaluation, MCC created a team which included the leadership, management, and the shop floor production operator. This versatile team led to different system viewpoints that collectively agreed on focusing to achieve the FR2: "Produce to customer-consumed quantity" and FR3: "Produce customer-consumed mix" out of the seven teaching FRs. Also, the company agreed on the importance of achieving FR5 that is about achieving FR1 through FR4 in spite of any variation (see Fig. 3). This collective understanding of the manufacturing system FRs led the team to develop a design decomposition focused on the pull system to support production to takt time, which would be effective for their company in order to meet the customer need of "on-time delivery of the demanded products." The analysis led to the top-level re-design FR-PS pair for the small bus: FR1: "Produce what is needed to ship today" PS1: "HVAC Small Bus Production System re-design". The re-design effort led to three major sub FRPS pairs. The areas of re-design and the chosen high-level solution along with sub FR-PS pairs are highlighted in the system design map of the HVAC small bus manufacturing system shown in Fig. 9. 


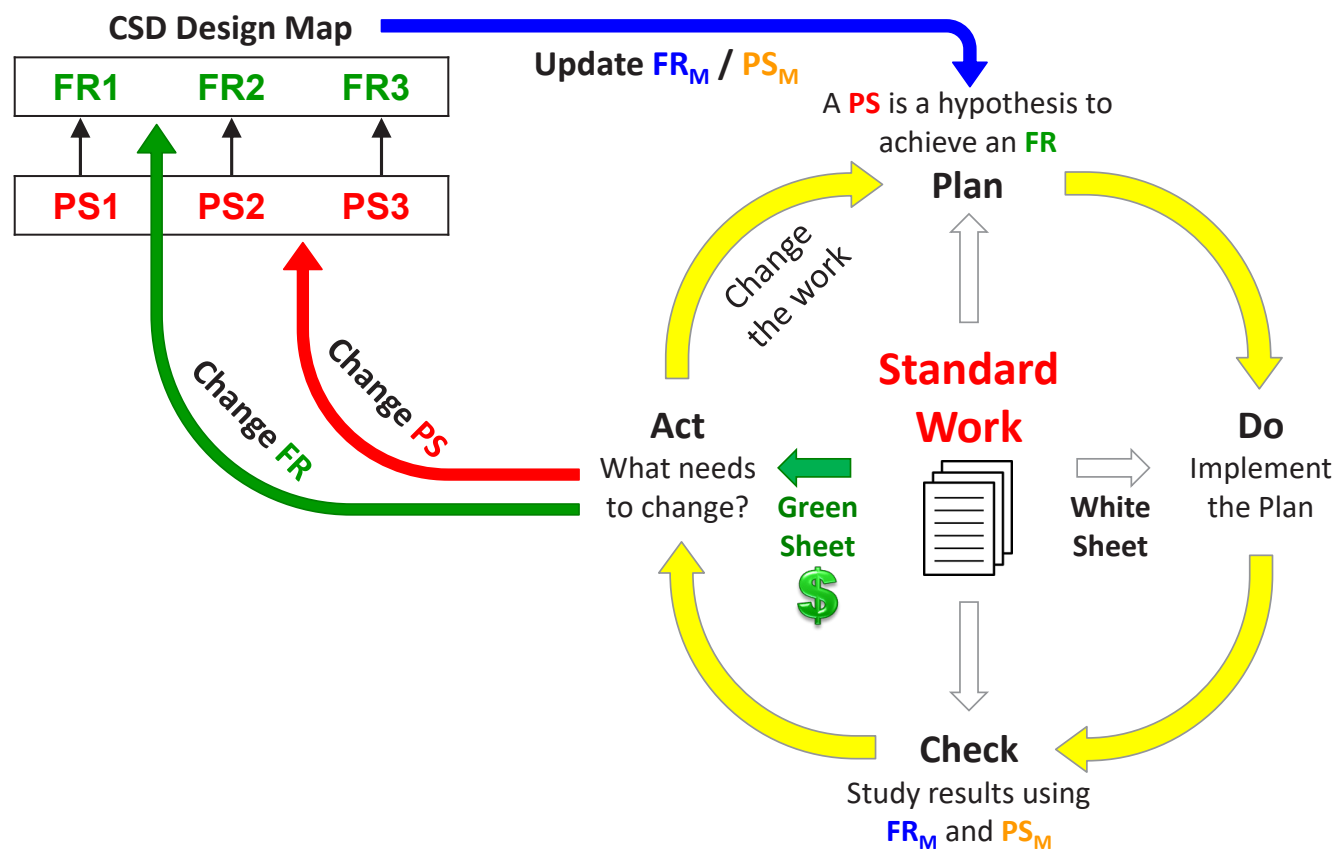

Figure 7. PDCA Cycle [19, 25]

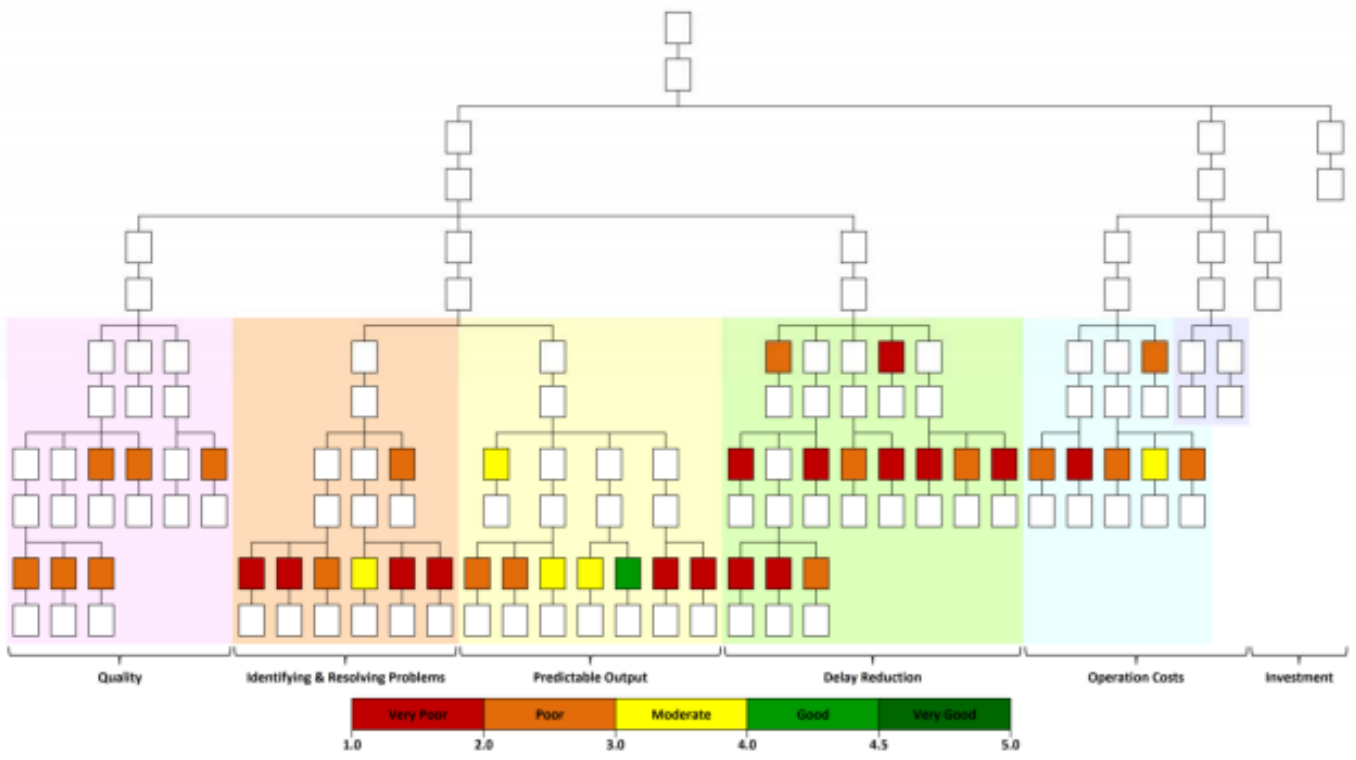

Figure 8. Analysis of Current System Design based on MSDD Evaluation

\subsection{Single-Piece Flow}

Collective agreement of team members upon deriving FRs from customer needs and choosing PSs for achieving those FRs was developed through physical simulation (Lego blocks) as shown in Fig. 10. The team collectively chose the PS: "Single-piece flow of production" for achieving the FR: "Reduce production delays" and simulated the production flow several times to balance the production lines to takt time of 6 mins 40 secs based on their average customer demand. The simulation also indicated that the single-piece flow of production was partially coupled with FR2 and FR3 of the decomposition in Fig. 9. The parameters chosen for the simulation are shown in Fig. 11

\subsection{Lean-linked Cell System Design}

In the past, there were several off-line stations called preparation units and still, the production lines could not produce to takt time because of system delays. The operators would work long hours to deal with increased production volume. Manufacturing cells were developed that integrated these off-line operations. Next, the lean linked cell system was designed by connecting the supplied parts, 


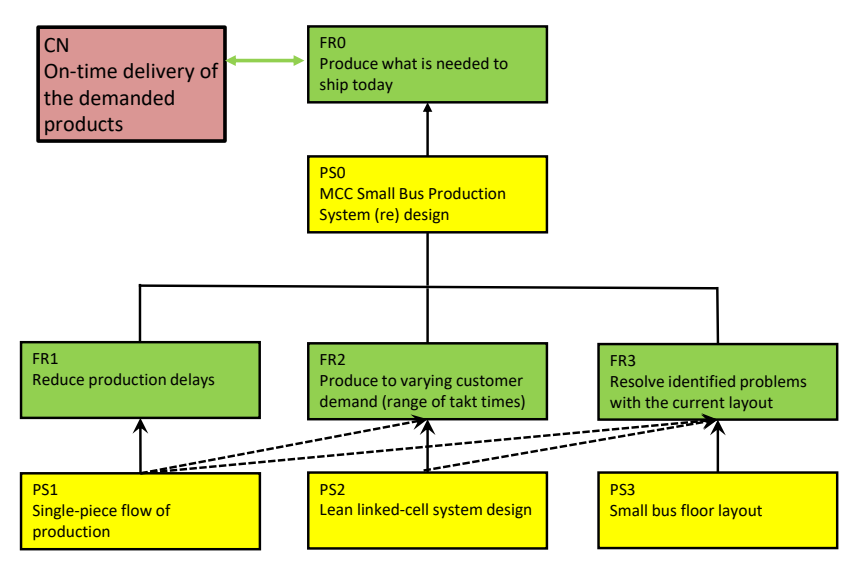

Figure 9. HVAC Manufacturing System Redesign on the Basis of MSDD Evaluation

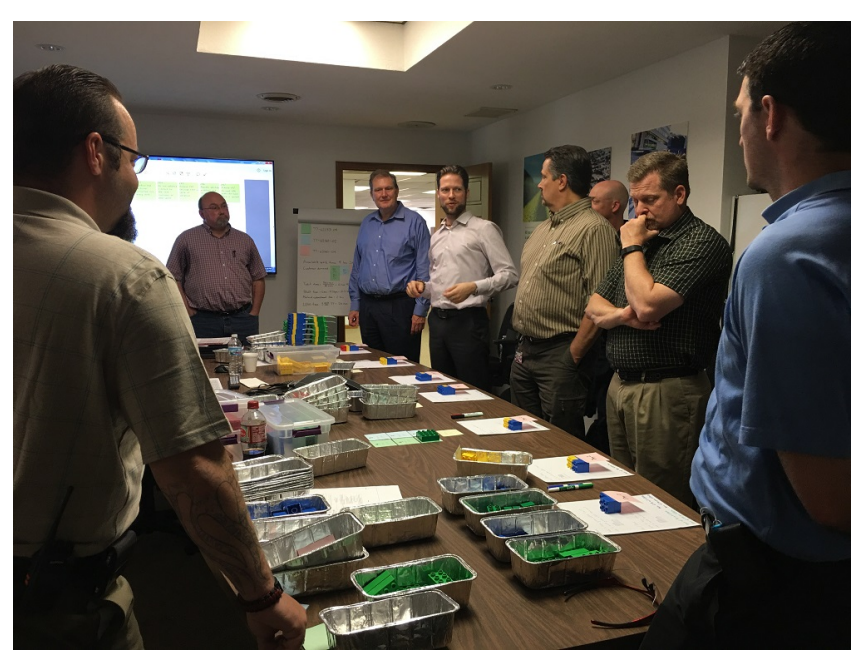

Figure 10. Collective Agreement of the Desired FR-PS pairs Using Physical Simulation (Figure used with permission from Mobile Climate Control)

assembly, and shipping with pull production information to achieve the FRs mentioned in the decomposition in the Fig. 12.

Standard work was designed for all the stations by balancing the operations to address changes in takt time. An increase/decrease in takt time was dealt with by re-arranging work tasks among the stations and by adding/removing a station to sustain the line balancing to takt time. Time studies were performed to balance the cycle time of each station $\left(C T_{i}\right)$ of the production lines to takt time as defined by Eq. 3 [38, 39].

$$
C T_{1} \approx C T_{2} \approx \ldots \approx C T_{n} \leq \text { Takt Time }
$$

Ergonomic station design was developed in order to reduce the cycle time of the stations by eliminating the unnecessary motions of turning back and forth for the parts at $180^{\circ}$. Besides, the potential for musculoskeletal disorders that used to result from picking the heavy parts off the floor were minimized.

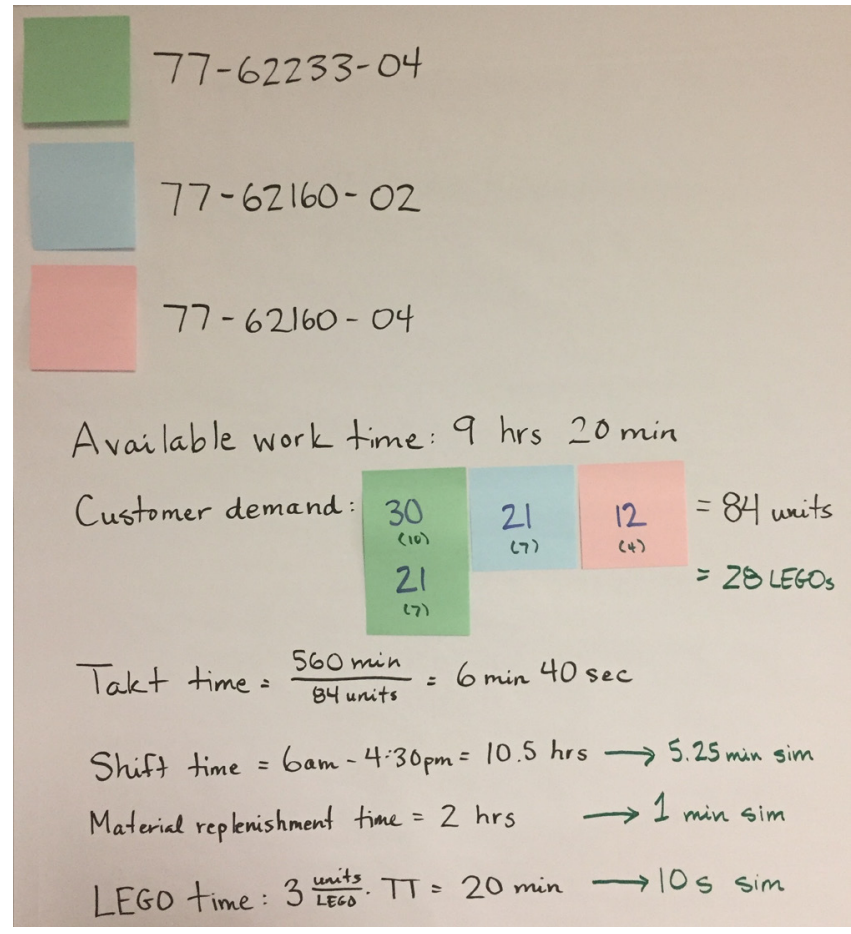

Figure 11. Simulation Parameters

For achieving the FR22: "Build any model at any time," the material inflow and outflow of smaller parts were located from the front to the station with an angle of inclination and declination of $15^{\circ}$ respectively. This approach of station re-design also helped the replenishment personnel to easily identify which bin is empty and which one is needed to be replaced. The pallets (larger parts) availability was assured at the right side of the station, as shown in Fig. 13.

The number of racks designed was according to the number of containers needed to hold all the material types and the number of rows in the flow-rack was designed on the basis of SWIP quantity. The height (90") of the flow rack and the length (30") of the working table was designed ergonomically to provide an easy access for the operator to the flow racks. Besides, the working-table height (35") was also designed according to the comfort level of the operator.

Standard Work In Process (SWIP) quantity for each part type at each station was ensured so that the production runs smoothly and all the parts are available at all times as opposed to the "zero inventory system." The calculation for SWIP quantity shown in Eq.s 4, 5, and 6. Let suppose,

$$
\begin{aligned}
& T T=\text { Takt time } \\
& n p=\text { parts needed per assembled unit (number/unit) } \\
& n c=\text { container size (number/container) } \\
& t d=\text { Time to detect (minutes) }
\end{aligned}
$$




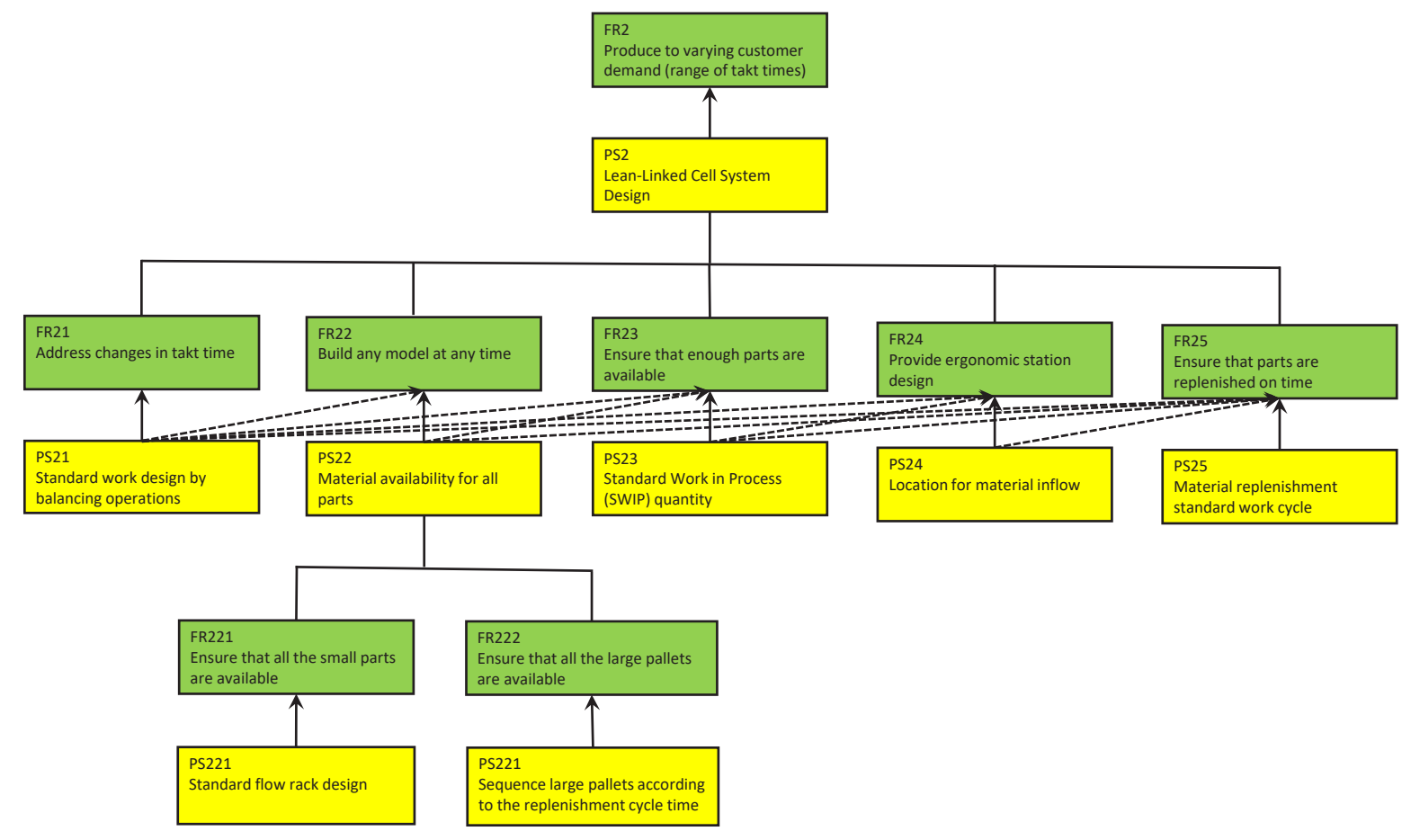

Figure 12. Lean-Linked Cell System Design Decomposition

$t r=$ Time to replenish (minutes)

$O R T=$ Operator Replenishment Time (minutes)

$F=$ Float needed/Emergency Work in Process (EWIP) (or uplift) needed (15 percent).

Where,

$$
\begin{gathered}
t d=t r \\
O R T=t d+t r
\end{gathered}
$$

Now, the formula developed for SWIP quantity is:

$$
S W I P=n p *(O R T) / T T *(1+F)
$$

The float percentage in the SWIP quantity is kept high in the beginning but is lowered down with improvement in the system predictability. This reduction of SWIP quantity is analogous to the reduction of medicine intake with health recovery.

A standard work cycle for the material replenishment was developed to ensure that parts are replenished on time to the operators, according to which the replenishment cycle time for smaller parts was set to two hours and for larger parts/pallets was set to one hour. The formula for the number of containers (nc) to be replenished after each standard work cycle is shown in Eq. 7:

$$
\text { Number of containers }=((S W I P+F) / n c)+1
$$

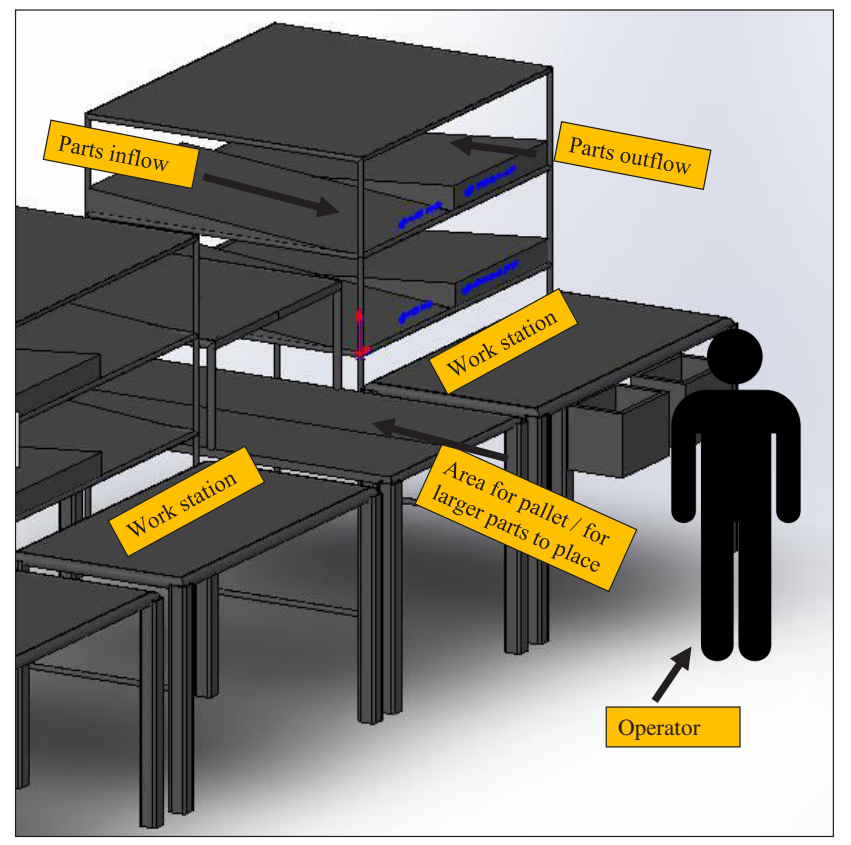

Figure 13. Ergonomic Station Design

\subsection{Revised Floor Layout}

The lean-linked cell system design led the team to agree on re-designing the line to ensure that enough space was available for production and assembly. The team came up with the idea of re-designing the lines in a way that operators would have to work inside the lines while the replenishment of the material would be done from back- 
side/outside of the lines as shown in Fig. 14. The team had incorporated the replenishment personnel and the working operators for collective agreement on the line re-design so that the operators would help implement the change rather than resist the change. This idea was brought up specifically from the replenishment personnel for the replenishment personnel knew the types of interference problems with the working operators that both of them used to encounter in the current system. Re-designing the lines resulted in saving the space of $\mathbf{2 1 0 0}$ square feet as an additional benefit to meeting the customer needs of quantity and mix of products. This saved space resulted in developing the designated aisles for material supply and finished goods, thereby eliminating the earlier issue of blocking the racks in the raw material warehouse with finished products to be shipped. Only finished product inventory meant to be shipped today would be stored in the dedicated lanes.

The planned system helped the plant in meeting the customer needs. The evaluation result at the end of the project depicted significant improvement indicated by the highlighted areas, as shown in Fig. 15.

\section{Conclusion}

Becoming lean means the system is intending to enact a system-wide change. That's why the CSD approach begins with the collective agreement of senior leadership to make a conscious decision to change (become lean). To enact change, there are several TPS tools available that can help assist a system to become lean. However, in order to become lean and sustain the long-term business sustainability, the system must address these three concerns: (1) Why should a tool(s) be implemented/used? (2) What sequence of implementing the tools ought to be followed? and (3) How to ensure customer needs are satisfied in a sustainable manner? The proposed approach, CSD, addresses the first concern by clarifying the intent behind using a tool(s) through its well-defined language that begins by understanding the internal and external customer needs prior to implementing a tool. The thinking layer of CSD (expressed as MSDD in this paper) deals with the FR-PS pair(s) for achieving customer needs with an understanding of the implementation sequence. The MSDD covers the customer needs by maximizing the quality of the product followed by minimizing the non-value added activities (resulting in reducing the mean time of the product in the system) and minimizing the long-term investment, which provides an implementation sequence for a system to result in becoming lean. The concern of the sustainability of a system design resulting in achieving customer needs is addressed by the work/actions layer of the CSD flame model. i.e., the employment of the PDCA cycle for continuous improvement with a consistent modification of the defined standard work in response to each improvement in the system design.

MCC system design was developed through a collective agreement process of all the people in the industry (leadership, management, operators) rather than just the leadership/consultants making the choices for change. This approach led to an easy replication of the small bus production system design to the rest of the facility. The collective agreement process also led the operators to assist in applying the change rather than resisting the change for their views were respected and valued during the system re-design development, thus resulting in sustainable systems.

\section{Acknowledgment}

A sincere thank you to the Mobile Climate Control team for working with the Center of Excellence in Systems Engineering at Purdue Fort Wayne, to apply the Collective System Design approach to design their manufacturing system to meet the customer needs. Also, a thank you is owed to Jason Barnes who is the Associate Director of the Center of Excellence in Systems Engineering at Purdue Fort Wayne. He assisted with simulation and mathematical analysis.

\section{References}

[1] M. Graban, The Term "Lean Production" is 25 Years Old - Some Thoughts on the Original John Krafcik Article (2013), www . leanblog.org

[2] P.M. Gibbons, S.C. Burgess, International Journal of Lean Six Sigma 1, 134 (2010)

[3] M. Imai, Kaizen: The Key To Japan's Competitive Success, 1st edn. (McGraw-Hill Education, New York, 1986), ISBN 978-0-07-554332-9

[4] J. Langstrand, The TQM Journal 21 (2009)

[5] T. Ohno, Toyota Production System; Beyond Large Scale Production (Productivity Press Portland, 1988)

[6] R.W. Hall, Zero Inventories (Dow Jones - Irwin, Homewood, IL, 1983), ISBN 978-0-87094-461-1

[7] D. Cochran, J. Swartz, Collective System Design of St. Francis Hospital ER (2015)

[8] J.P. Womack, D.T. Jones, D. Roos, The Machine That Changed the World (Free Press, 1990), ISBN 978-07432-9979-4

[9] S. Mostafa, J. Dumrak, H. Soltan, Production \& Manufacturing Research 1, 44 (2013)

[10] Sweta, IPEDR 75.26, 127 (2014)

[11] G. Dulhai, Management \& Marketing 3 (2008)

[12] P. Arunagiri, A. Gnanavelbabu, Procedia Engineering 97, 2072 (2014)

[13] D. Cochran, M. Kawada, Education Approach in Japan for Management and Engineering of Systems (San Antonio, 2012)

[14] D.S. Cochran, S. Hendricks, J. Barnes, Z. Bi, Journal of Manufacturing Science and Engineering 138, 101006 (2016)

[15] D.S. Cochran, in Lean Accounting (WileyBlackwell, 2015), pp. 263-297, ISBN 978-1119-19680-8

[16] J. Womack, "Keynote Presentation" (2011)

[17] D.S. Cochran, Systems Approach to Sustain Lean Organizations (Cobo Hall, Detroit, MI, 2007) 


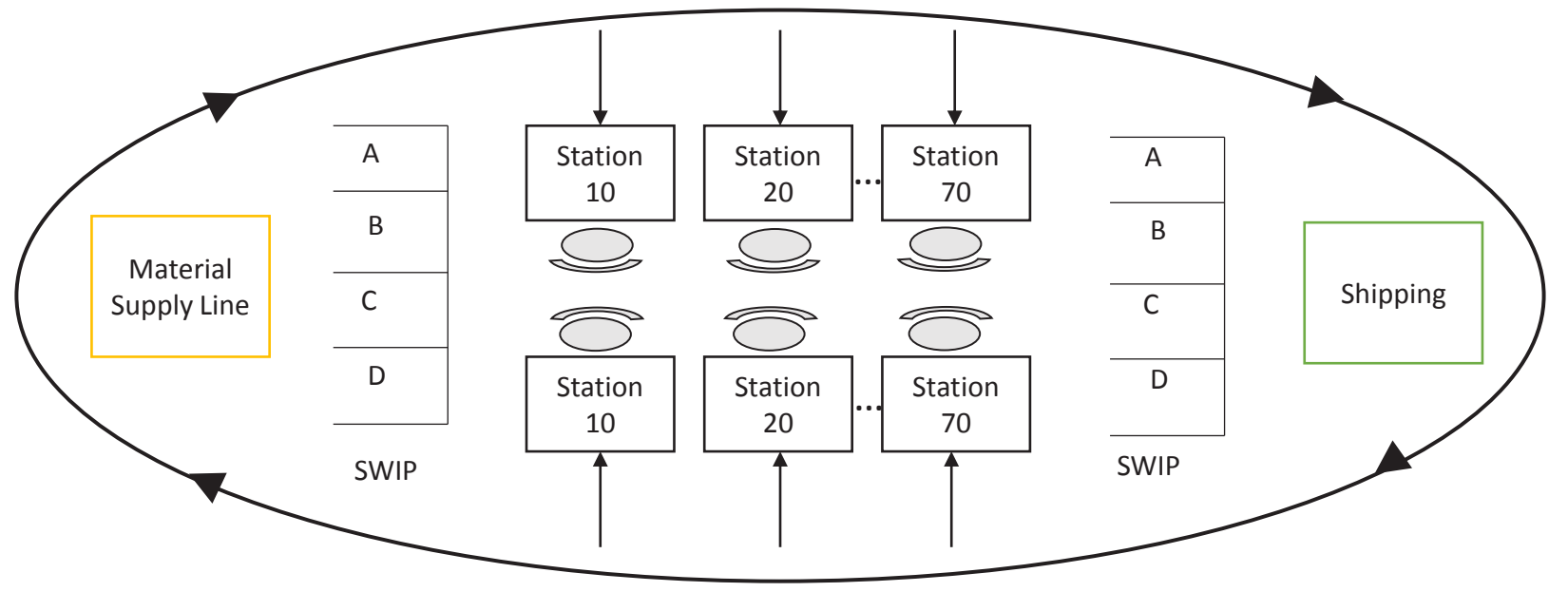

Standard Replenishment Person Cycle

Figure 14. Re-designed Line Layout Achieved Through Collective Agreement

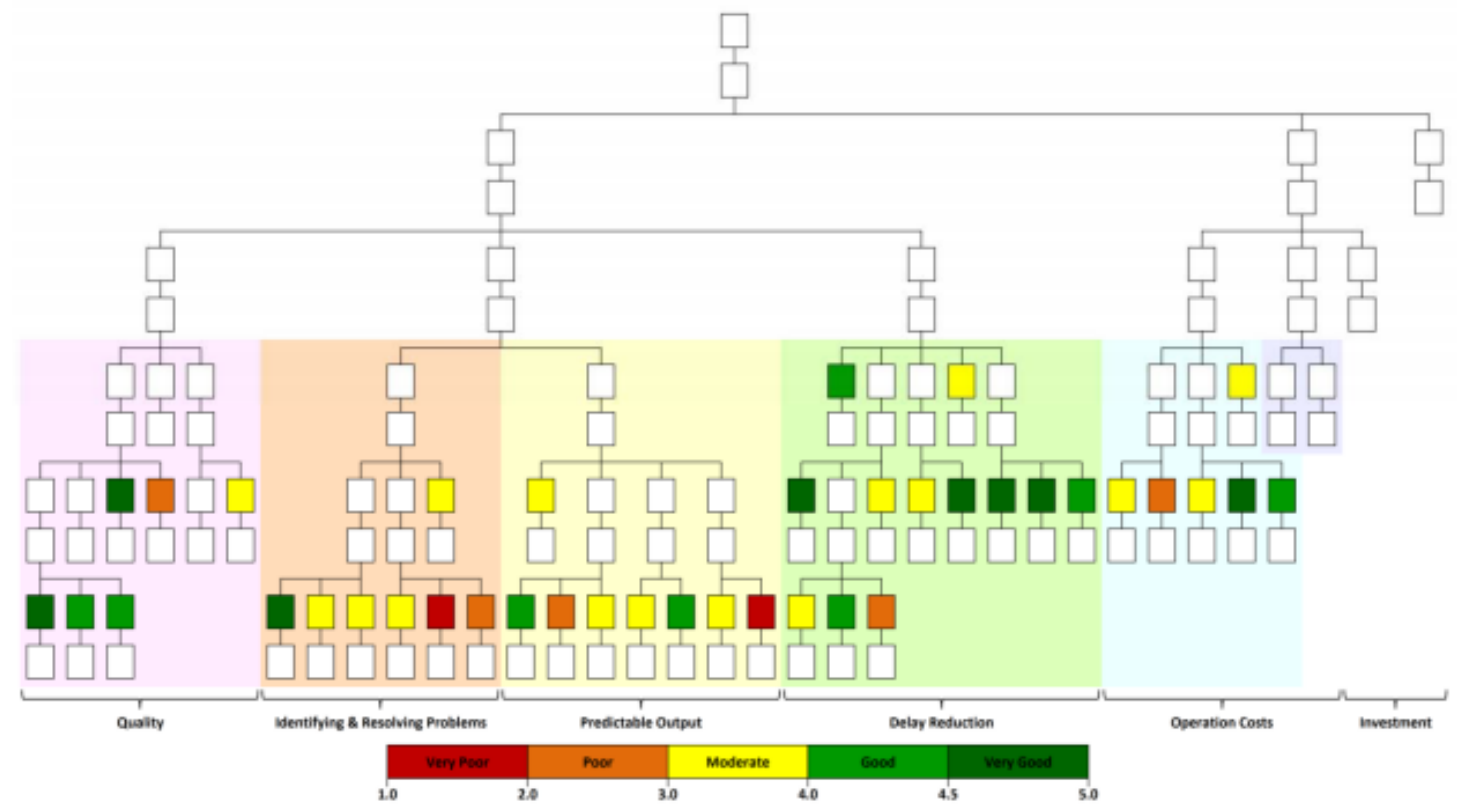

Figure 15. Analysis of Planned System Design based on MSDD Evaluation

[18] Why lean doesn't work? (Industrial Solution Inc., 2014), p. 62

[19] D.W. Deming, Out of the Crises, 1st edn. (The MIT Press, 2000), ISBN 0-262-54115-7

[20] J. Salatin, You Can Farm, 1st edn. (Polyface, 1998)

[21] D. Cochran, J. Barnes, Collective System Design (CSD) in sustaining the lean enterprise (2013)

[22] Taiichi Ohno Quotes Help Drive Lean Culture and Acceptance (2008), http: //matthrivnak.com

[23] Commissioner, Consumer Updates - Avoiding Drug Interactions, https://www.fda.gov/ ForConsumers/ConsumerUpdates/ucm\$96386. htm
[24] V.E. Emiel, P. Pollet, Did Toyota fool the lean community for decades? (2014), https://www.slideshare.net/EmielVanEst/ did-toyota-fool-the-lean-community-for-decades/ 79-Emiel_van_EstWhere_there_is

[25] D.S. Cochran, R. Sereno, W. Aldrich, p. 12 (2014)

[26] D.S. Cochran, System design; lean manufacturing; tps (2007), http://systemdesignllc.com/ about.html

[27] S. Nam-pyo, Complexity: Theory and Applications (Oxford University Press, 2005), ISBN Oxford University Press 
[28] D.S. Cochran, J.J. Smith, Procedia Manufacturing 26, 1369 (2018)

[29] A. Spencer, D. Cochran, Procedia CIRP 34, 143 (2015)

[30] J. Linck, D. Cochran, The Importance of Takt Time in Manufacturing System Design (1999)

[31] J. Womack, J. Shook, Gemba Walks, expanded 2nd edition edn. (Lean Enterprises Inst Inc, 2013), ISBN 978-1-934109-38-0

[32] D. Cochran, Annual Conference \& Exposition pp. 15.287.1-15.287.12 (2010)

[33] D.S. Cochran, Y.S. Kim, J. Kim, The Impact of Performance Measurement on Manufacturing system design (Cambridge, MA, 2000), pp. 1-8
[34] D.S. Cochran, J.F. Arinez, J.W. Duda, J. Linck, Journal of Manufacturing Systems 20, 371 (2001)

[35] A. Simboli, R. Taddeo, A. Morgante (2014)

[36] W.J. Hopp, M.L. Spearman, Factory Physics: Foundations of Manufacturing Management, first edition edition edn. (Richard D Irwin, Chicago, 1995), ISBN 978-0-256-15464-1

[37] N.K. Shimbun, H. Hirano, Poka-Yoke: Improving Product Quality by Preventing Defects (Productivity Press Portland, 1988), ISBN 0-915299-31-3

[38] D. Cochran, J. Foley, B. Zhuming, pp. 870-890 (2016)

[39] D.S. Cochran, D.C. Dobbs, Journal of Manufacturing Systems 20, 390 (2001) 